\title{
QUAL A CONTRIBUIÇÃO DAS CARACTERÍSTICAS ANTROPOMÉTRICAŞ NA VELOCIDADE DE CORRIDA DE CURTA DISTÂNCIA?
}

\author{
Diego de Alcantara Borba \\ Universidade do Estado de Minas Gerais, Ibirité, Minas Gerais, Brasil
}

\author{
João Batista Ferreira-Júnior \\ Gerais, Brasil \\ Valdênio Martins Brant \\ Universidade Federal de Minas Gerais, Belo Horizonte, Minas Gerais, Brasil

\section{Juliana Bohnen Guimarães} \\ Universidade do Estado de Minas Gerais, Ibirité, Minas Gerais, Brasil \\ Carlos Alexandre Vieira \\ Universidade Federal de Goiás, Goiânia, Goiás, Brasil
}

Instituto Federal de Educação, Ciência e Tecnologia do Sudeste de Minas Gerais, Rio Pomba, Minas

\begin{abstract}
Resumo
O estudo buscou avaliar os efeitos das características antropométricas na velocidade de corrida. Foram avaliados a estatura, massa corporal (MC), índice de massa corporal (IMC), dobra cutânea panturrilha $\left(\mathrm{DC}_{\mathrm{pa}}\right)$, comprimento da tíbia $\left(\mathrm{C}_{\mathrm{tibia}}\right)$ e velocidade da corrida $(50 \mathrm{~m})$ de 235 estudantes. A análise de regressão múltipla explicou $62 \%$ do desempenho na corrida $(\mathrm{p}<0,05)$. A análise de regressão linear simples mostrou que a $\mathrm{DC}_{\mathrm{pa}}$ correspondeu a $45 \%$, estatura $33 \%, \mathrm{C}_{\mathrm{tibia}} 24 \%$ e MC $3 \%$, do desempenho na corrida $(p<0,05)$. A análise de cluster mostrou que o grupo de baixa velocidade apresentava maior $\mathrm{MC}$, IMC e $\mathrm{DC}_{\mathrm{pa}}(\mathrm{p}<0,05)$, e o grupo de alta velocidade apresentava maior estatura, $\mathrm{C}_{\text {tibia }}$ e menor $\mathrm{DC}_{\mathrm{pa}}(\mathrm{p}<0,05)$. Conclui-se que as medidas antropométricas podem predizer o desempenho da corrida. Palavras chave: Velocidade. Corrida. Antropometria. Desempenho.
\end{abstract}

\section{Introdução}

O desempenho em uma corrida de curta distância depende da otimização das fases de: 1) aceleração, 2) manutenção da velocidade máxima e 3) desaceleração (Mackala, 2007). Tem sido reportado que a interação entre a amplitude e a frequência de passada determina o desempenho, como um todo ou em fases específicas, de corridas de curta distância (Hunter et al., 2004; Graubner, 2011). O aumento de ambas as variáveis simultaneamente é difícil, devido a dependência inversa entre essas variáveis (Hunter et al., 2004; Mackala, 2007). Desse modo, o aumento em uma destas variáveis e a manutenção da outra pode resultar em melhoria da velocidade da corrida (Mackała, 2013).

Características antropométricas como a estatura e comprimento da perna do indivíduo podem afetar desempenho de tarefas que exigem o deslocamento do corpo em alta velocidade e curta distância. A possível vantagem de uma maior estatura e comprimento das pernas podem estar associados a maior amplitude da passada (Mackała, 2013; Mackala et al., 2015). Assim, para um mesmo número de passadas, sujeitos com maior estatura e comprimento das pernas conseguiriam percorrer maior distância quando comparados a sujeitos com menor 
estatura e comprimento das pernas. Num estudo com 11 corredores profissionais, foi encontrada correlação positiva entre o comprimento da perna e a velocidade de corrida de 10 $\mathrm{m}(\mathrm{r}=0,84)$ e $30 \mathrm{~m}(\mathrm{r}=0.88)$ (Mackala et al., 2015). A maior estatura de Usain Bolt $(1,96 \mathrm{~m})$, atual recordista mundial dos $100 \mathrm{~m}$ rasos $(9,58 \mathrm{~s})$, comparado à estatura dos ex-recordistas mundiais, Tyson Gay $(1,83 \mathrm{~m})$ e Asafa Powell $(1,90 \mathrm{~m})$, tem chamado atenção tanto da mídia quanto da comunidade científica (Thomas et al., 1992). Em um estudo descritivo das características físicas dos finalistas dos $100 \mathrm{~m}$ rasos dos Jogos Olímpicos de 2012, identificou-se que Usain Bolt é quase $20 \mathrm{~cm}$ mais alto que a média dos outros competidores (Mackała, 2013). Sendo assim, a maior estatura de Bolt pode estar relacionada também a um maior comprimento de passada, e portanto, maior eficiência de corrida.

Outras duas variáveis que podem interferir na velocidade da corrida são a massa corporal e sua distribuição entre massa gorda e magra. Nesse sentido, Perez-Gomez et al. (Perez-Gomez et al., 2008) realizaram análise de regressão para determinar a contribuição de algumas características de composição corporal na velocidade de corrida de $30 \mathrm{~m}$ em 23 jogadores de handebol de ambos os sexos. Os resultados mostraram correlação positiva entre a massa muscular magra dos membros inferiores e a potência pico no teste de Wingate e com o tempo de corrida de $30 \mathrm{~m}$. Já o percentual de gordura correlacionou negativamente com o desempenho de corrida de $30 \mathrm{~m}$. Do ponto de vista de eficiência, quanto maior a massa corporal, maior o gasto de energia para seu deslocamento. O resultado seria movimentos mais lentos, principalmente se o excesso for representado pela gordura corporal, visto que, o tecido adiposo não é capaz de gerar tensão para a realização do movimento. Por outro lado, uma maior massa corporal poderia estar associada a maiores valores de massa muscular, o que levaria a maior velocidade de corrida, já que esta capacidade física está diretamente associada à área de secção transversa do músculo e consequentemente com maior potência muscular (Hunter et al., 2004). Usain Bolt apresentou-se 14.8\% mais pesado em Pequim, $12.3 \% \mathrm{em}$ Berlin, e 10.7\% em Londres que os demais finalistas dos $100 \mathrm{~m}$ rasos (Mackała, 2013). Portanto, uma quantidade ótima de massa corporal pode ser um fator determinante no desenvolvimento da velocidade máxima, afetando o desempenho em corrida de curta distância.

Entretanto, ainda não se sabe o quanto estas características antropométricas (i.e., massa corporal, dobra cutânea da panturrilha, estatura e comprimento da tíbia) podem determinar a velocidade em corrida de curta distância, em jovens pós-púberes. Considerando que uma das principais preocupações de técnicos, preparadores físicos, treinadores e professores de educação física é identificar parâmetros que contribuam para explicar a performance em diversas modalidades esportivas, estas informações poderão ser úteis para identificar características físicas associadas ao desempenho em corridas que exijam máxima velocidade. Além disso, essas informações poderão ser utilizadas como parâmetro alternativo para predizer o desempenho. Sendo assim, o objetivo do presente estudo é determinar o quanto a estatura, a massa corporal, o comprimento da tíbia e a dobra cutânea da panturrilha podem determinar juntas e separadamente o desempenho na corrida de $50 \mathrm{~m}$ em jovens póspúberes (17 e 18 anos) de ambos os sexos. Por se tratar de indivíduos não atletas, é esperado que uma menor massa corporal $(\mathrm{MC})$ e dobra cutânea da panturrilha $\left(\mathrm{DC}_{\mathrm{pa}}\right)$, e maior estatura e comprimento da tíbia $\left(\mathrm{C}_{\text {tíbia }}\right)$ estejam associadas com o maior desempenho na corrida de 50 m.

\section{Material e métodos}

O tamanho da amostra foi determinando pelo uso do software GPower (versão 3.1.2; Franz Faul, Universitat Kiel, Alemanha), considerando as seguintes especificações: tamanho do efeito $f^{2}=0.15 ; \alpha=0.05 ;(1-\beta)=0.8$; número de preditores $=5$; família do teste $=\mathrm{F}$ teste e 
teste estatístico = análise de regressão linear múltipla- modelo fixo, desvio $\mathrm{R}^{2}$ a partir de zero (Back, 2013). O tamanho da amostra estimado de acordo com essas especificações foi de 92 sujeitos. Participaram do estudo 235 jovens de ambos os sexos, entre 17 e 18 anos de idade. Os voluntários eram alunos do ensino médio e superior de uma escola federal de Belo Horizonte, Minas Gerais, Brasil. Os critérios de inclusão foram; a) participar regularmente das aulas de educação física (duas aulas semanais com duração total de 100 min), b) praticar regularmente atividades esportivas ou recreativas que requerem corridas de curta distância e alta velocidade, c) ter entre 17 e 18 anos de idade, d) responder "não" a todas as perguntas do questionário para prontidão ao exercício físico Par-Q (Thomas et al., 1992). Os voluntários e ou responsáveis assinaram um termo de Consentimento Livre e Esclarecido. Este estudo respeitou as normas do Conselho Nacional de Saúde e Pesquisa com seres humanos (466/12), sendo aprovado pelo comitê de ética em pesquisa da Universidade do Estado de Minas Gerais, sob o parecer número 1.306.982.

Para realização do estudo os voluntários visitaram o laboratório dois dias. No primeiro dia os voluntários foram instruídos quanto aos procedimentos do estudo e foi entregue o temor de Consentimento Livre e Esclarecido. No segundo dia foram realizadas as medidas antropométricas e a corrida de $50 \mathrm{~m}$. As medidas antropométricas foram realizadas na seguinte ordem: massa corporal (MC), estatura, dobra cutânea da panturrilha $\left(\mathrm{DC}_{\mathrm{pa}}\right)$ e comprimento da tíbia $\left(\mathrm{C}_{\text {tibia }}\right)$. Em seguida os voluntários realizaram duas corridas de $50 \mathrm{~m}$ com 10 min de intervalo entre as corridas. As medidas antropométricas e as duas corridas de $50 \mathrm{~m}$ foram conduzidas sempre pelo mesmo avaliador. Os procedimentos foram realizados durante as aulas de educação física no período entre 14 e $16 \mathrm{~h}$.

Ao sinal do primeiro apito do pesquisador, o voluntário se posicionava atrás da linha de partida em posição de expectativa. Ao sinal do segundo apito, o voluntário corria a distância de $50 \mathrm{~m}$ no menor tempo possível. O cronometro era iniciado junto ao segundo apito e travado quando o tronco do voluntário cruzava a linha dos $50 \mathrm{~m}$. O teste foi realizado em uma pista de $250 \mathrm{~m}$. O menor tempo encontrado em duas tentativas foi utilizado para o cálculo da velocidade da corrida (VC). A VC foi estimada dividindo-se a distância percorrida $(50 \mathrm{~m})$ pelo tempo gasto.

A MC e a estatura foram medidas usando uma balança acoplada a um estadiômetro (Filizola ${ }^{\circledR}$, Brasil). A MC e a estatura foram medidas com o voluntário descalço, usando short e camisa de manga curta. Durante a medição da MC e estatura, os voluntários permaneceram imóveis olhando para o horizonte. A $\mathrm{DC}_{\mathrm{pa}}$ foi medida com uso de adipômetro (Cescorf ${ }^{\circledR}$, EUA). $\mathrm{O}$ avaliador posicionou o adipômetro na região medial da panturrilha direita mantendo a dobra posicionada com uma das mãos. A $\mathrm{C}_{\text {tíbia }}$ foi medida utilizando uma fita antropométrica posicionada ao centro do maléolo medial e estendida até o centro da patela. Para a medição da $\mathrm{DC}_{\mathrm{pa}}$ e da $\mathrm{C}_{\text {tíbia, }}$ os voluntários permaneceram de pé, com o pé direito sobre uma cadeira de modo que a articulação do joelho formasse um ângulo de aproximadamente $90^{\circ}$.

Os dados foram apresentados como média e desvio padrão. A normalidade dos dados foi garantida após a realização do teste de Kolmogorov-Smirnov. Para avaliar o grau de determinação das variáveis antropométricas sobre a velocidade da corrida foi realizada a análise de regressão linear múltipla. Além disso, foi realizada a análise de regressão linear simples para determinação da participação de cada uma das variáveis antropométricas. Uma análise de cluster de duas etapas foi usada para criar grupos homogéneos de acordo com as variáveis medidas. Uma ANOVA one-way foi utilizada para comparar os grupos criados nas seguintes variáveis: $\mathrm{VC}$, estatura, $\mathrm{MC}, \mathrm{IMC}, \mathrm{DC}_{\mathrm{pa}}$ e $\mathrm{C}_{\text {tíbia. }}$. Em caso de diferença significativa foi utilizado o teste post-hoc de Tukey. O nível de significância adotado foi de $5 \%$. Valores de $f^{2}$ foram calculados de acordo com Beck ${ }^{(1)}$. Valores de $f^{2}$ de $0,02,0,15$ e 0,35 foram utilizados para definir o tamanho do efeito $f^{2}$ como pequeno, médio e grande, respectivamente ${ }^{(1)}$. 


\section{Resultados}

A tabela 1 mostra os dados descritivos das características antropométricas e o desempenho na corrida de $50 \mathrm{~m}$ dos voluntários.

Tabela 1: Dados antropométricos e velocidade de corrida

\begin{tabular}{|c|c|c|c|c|c|}
\hline \multicolumn{6}{|c|}{ Variáveis $(\mathbf{n}=\mathbf{2 3 5})$} \\
\hline Estatura (m) & $\mathrm{MC}(\mathrm{kg})$ & IMC $\left(\mathrm{kg} \cdot \mathrm{m}^{-2}\right)$ & $\mathrm{DC}_{\mathrm{pa}}(\mathrm{mm})$ & $\mathrm{C}_{\text {tíbia }}(\mathrm{cm})$ & $\mathrm{VC}\left(\mathrm{m} \cdot \mathrm{s}^{-1}\right)$ \\
\hline $1,7 \pm 0.1$ & $58,2 \pm 11,3$ & $21,3 \pm 3,7$ & $20,8 \pm 8,8$ & $37,9 \pm 2,7$ & $5,7 \pm 0,7$ \\
\hline
\end{tabular}

Com a análise de regressão linear múltipla foi encontrado um coeficiente de determinação de 0,621 (poder da amostra $=1,0, \mathrm{p}<0.05$ ). Este resultado indica que as variáveis antropométricas, juntas, explicam aproximadamente $62 \%$ da $\mathrm{VC}(\mathrm{p}<0,05)$ com o tamanho do efeito considerado como grande $\left(f^{2}=1,61\right)$. A contribuição de cada variável foi determinada através da regressão linear simples. Os resultados mostraram que $\mathrm{DC}_{\mathrm{pa}}$ corresponde a $45 \%$, a estatura $33 \%$, a $\mathrm{C}_{\text {tíbia }} 24 \%$ e a MC a apenas $3 \%$ da velocidade da corrida de $50 \mathrm{~m}(\mathrm{p}<0,05)$. $\mathrm{O} \mathrm{C}_{\text {tíbia }}$ e a estatura apresentaram relação positiva com a $\mathrm{VC}$, enquanto que a $\mathrm{DC}_{\mathrm{pa}}$ relacionou de modo negativo com a VC (Tabela 2). O IMC não apresentou relação com a VC $(p>0.05)$.

Tabela 2: Coeficiente de determinação $(\mathrm{R})$ e coeficiente de determinação ao quadrado $\left(\mathrm{R}^{2}\right)$ das regressões múltipla e linear.

\begin{tabular}{lccc}
\hline \multicolumn{1}{c}{ Variáveis } & $\mathbf{R}$ & $\mathbf{R}^{2}$ & $\mathbf{p}$ \\
\hline VC+Estatura+MC+IMC+DC $+\mathrm{pa}_{\mathrm{p}}+\mathrm{C}_{\text {tibia }}$ & 0,788 & 0,621 & $<0,001^{*}$ \\
VC+Estatura & 0,576 & 0,332 & $<0,001^{*}$ \\
$\mathrm{VC}+\mathrm{MC}$ & 0,179 & 0,0319 & $0,006^{*}$ \\
$\mathrm{VC}+\mathrm{IMC}$ & 0,096 & 0,0093 & 0,139 \\
$\mathrm{VC}^{-D C}$ & $-0,672$ & 0,451 & $<0,001^{*}$ \\
$\mathrm{VC}_{\mathrm{pa}}$ & 0,482 & 0,230 & $<0,001^{*}$
\end{tabular}

(*) Coeficiente de determinação significativo. $\mathrm{MC}=$ massa corporal; $\mathrm{IMC}=$ índice de massa corporal; $\mathrm{DC} \mathrm{pa}_{\mathrm{pa}}=$ dobra cutânea da panturrilha; $\mathrm{C}_{\mathrm{t} \text { tibia }}=$ comprimento da tíbia; $\mathrm{VC}=$ velocidade da corrida.

A análise de cluster mostrou três grupos: 1) Baixa velocidade $(\mathrm{n}=56), 2)$ Média velocidade $(n=91)$ e 3$)$ Alta velocidade $(n=88)$ (Tabela 3$)$. Foi observada diferença entre os grupos nas variáveis $\mathrm{VC}(\mathrm{F}=158,9, \mathrm{p}<0,001)$, estatura $(\mathrm{F}=151,5, \mathrm{p}<0,001), \mathrm{MC}(\mathrm{F}=101,3$, $\mathrm{p}<0,001), \mathrm{IMC}(\mathrm{F}=36,3, \mathrm{p}<0,001), \mathrm{DC}_{\mathrm{pa}}(\mathrm{F}=143,8, \mathrm{p}<0,001)$ e $\mathrm{C}_{\text {tibia }}(\mathrm{F}=75,8, \mathrm{p}<0,001) . \mathrm{O}$ grupo de baixa velocidade foi composto pelos sujeitos com a maior MC ( $\mathrm{p}<0,05)$, IMC ( $\mathrm{p}<$ $0,05)$ e $\mathrm{DC}_{\mathrm{pa}}(\mathrm{p}<0,05)$. Por outro lado, o grupo de alta velocidade foi formado pelos sujeitos com a maior estatura $(\mathrm{p}<0,05)$ e $\mathrm{C}_{\text {tibia }}(\mathrm{p}<0,05)$, e a menor $\mathrm{DC}_{\mathrm{pa}}(\mathrm{p}<0,05)$. 
Tabela 3: Média e desvio padrão dos três grupos criados pela análise de cluster.

\begin{tabular}{|c|c|c|c|c|c|c|}
\hline Grupos & $\mathrm{VC}\left(\mathrm{m} \cdot \mathrm{s}^{-1}\right)$ & $\begin{array}{c}\text { Estatura } \\
(\mathbf{m})\end{array}$ & MC (kg) & $\begin{array}{c}\text { IMC } \\
\left(\mathbf{k g} / \mathbf{m}^{2}\right)\end{array}$ & $\mathrm{DC}_{\mathrm{pa}}(\mathrm{mm})$ & $\mathrm{C}_{\text {tíbia }}(\mathrm{cm})$ \\
\hline $\begin{array}{c}\text { Baixa } \\
\text { velocidade } \\
(\mathrm{n}=56)\end{array}$ & $5,13 \pm 0,42$ & $1.62 \pm 0.06$ & $66,35 \pm 9,90$ & $24,43 \pm 5.51$ & $31,28 \pm 5,99$ & $37,21 \pm 2,89$ \\
\hline $\begin{array}{l}\text { Média } \\
\text { velocidade } \\
(\mathrm{n}=91)\end{array}$ & $5,34 \pm 0,47 *$ & $1.59 \pm 0.04 *$ & $50,15 \pm 4,42 *$ & $19,74 \pm 1,77^{*}$ & $21,26 \pm 5,95^{*}$ & $36,18 \pm 1,67 *$ \\
\hline $\begin{array}{c}\text { Alta } \\
\text { velocidade } \\
(\mathrm{n}=88)\end{array}$ & $6,32 \pm 0,32 * \#$ & $1.72 \pm 0,04 * \#$ & $62,44 \pm 8,04 * \#$ & $20.93 \pm 2.50$ *\# & $14,61 \pm 5,36^{* \#}$ & $40,02 \pm 1,97$ *\# \\
\hline
\end{tabular}

\section{Discussão}

O objetivo do presente estudo foi quantificar a contribuição das características antropométricas sobre a velocidade de corrida de curta distância em jovens não atletas, visto que, o papel das características antropométricas no desempenho da velocidade de corrida em jovens tem recebido limitada atenção. Era esperado que uma maior VC estivesse associada com menor $\mathrm{MC}$ e $\mathrm{DC}_{\mathrm{pa}}$, e maior estatura e $\mathrm{C}_{\text {tíbia. }}$ Os resultados confirmaram esta hipótese, já que foi observado que o grupo de alta velocidade de corrida apresentou a menor $\mathrm{MC}, \mathrm{DC} \mathrm{pa}$, IMC e maior estatura e $\mathrm{C}_{\text {tíbia }}$. Além disso, $62 \%$ do desempenho da corrida de jovens póspúberes pode ser explicado pelas variáveis antropométricas medidas. $\mathrm{A}_{\mathrm{DC}}$ determinou $45 \%$ da variação da velocidade, seguida da estatura, com $33 \%$ e C $\mathrm{C}_{\text {tíbia }}$ com $24 \%$.

Sabe-se que a velocidade da corrida é influenciada pela amplitude e frequência de passada (Hunter et al., 2004; Mackala, 2007). O comprimento da passada é determinado pelo comprimento do membro inferior e pela força desenvolvida pelos músculos extensores do quadril, joelho e tornozelo, durante a fase de contato com o solo (Coh et al., 2001). Assim, sujeitos com amplitudes maiores cobririam maior distância para um mesmo número de passadas comparado a sujeitos com menor amplitude. Por outro lado, grandes amplitudes normalmente estão associadas a menores frequências de passadas (Hunter et al., 2004). Valores mais altos de frequência de passada são mais facilmente alcançados com menor comprimento do membro inferior, devido ao menor braço de momento de força (Nagano e Komura, 2003). Assim, uma máxima velocidade de corrida é determinada pela relação ótima entre comprimento e frequência da passada e não somente pela magnitude de uma delas (Nagano e Komura, 2003; Hunter et al., 2004). Essa relação entre o comprimento e frequência da passada pode explicar a contribuição positiva da maior estatura e $\mathrm{C}_{\text {tíbia }}$ sobre a velocidade da corrida observada no corrente estudo. Outros estudos corroboram esta hipótese. Guedes e Guedes encontraram correlação significativa entre o desempenho no teste de corrida de $50 \mathrm{~m}$ e a estatura em 1180 escolares de ambos os sexos dos sete aos 17 anos (masculino $r=0,81$; feminino $r=0,69$ ) (Guedes e Guedes, 1993). Maćkała, Fostiak e Kowalski também verificaram que a velocidade de corrida de 10 e $30 \mathrm{~m}$ correlacionaram positivamente com o comprimento da perna ( $\mathrm{r}=0,84$ e $\mathrm{r}=0,88$, respectivamente) em velocistas com média de idade de 21 anos (Mackala et al., 2015).

Era esperado que a VC diminuísse à medida que MC aumentasse, pois se tratava de uma amostra de não atletas, no qual a quantidade de gordura em relação ao peso corporal total é provavelmente maior que em corredores de profissionais, os quais apresentam valores elevados de MC, porém como resultado de uma elevada massa muscular. Sabe-se que a potência produzida por um determinado músculo está associada à sua área de secção transversa e que o desempenho na corrida de $50 \mathrm{~m}$ depende, em grande parte, da capacidade 
de gerar força em um espaço curto de tempo (Ross e Leveritt, 2001; Ross et al., 2001; PerezGomez et al., 2008). Portanto, grande quantidade de gordura corporal pode ter prejudicado a eficiência do grupo baixa velocidade em produzir alta velocidade de corrida. Além disso, a maior quantidade de gordura na panturrilha contribuiria para seu maior peso e consequentemente afetaria o momento de força durante a corrida, fazendo com que o corredor tenha que exercer mais força para movimentar a perna durante a corrida, resultando na redução da sua velocidade. Foi verificado no atual estudo que jovens com maiores valores de $\mathrm{DC}_{\mathrm{pa}}$ foram mais lentos. Provavelmente a gordura localizada na panturrilha atuou como um "peso extra" a ser transportada ao longo da corrida. Sendo assim, parece que a $\mathrm{DC}_{\mathrm{pa}}$ é uma medida capaz de substituir avaliações mais complexas para determinar os efeitos da gordura corporal sobre a VC.

Os achados de Perez-Gomez et al. (Perez-Gomez et al., 2008) e Ré et al. (Ré, 2005) suportam esta hipótese. Perez-Gomez et al. encontraram que o percentual de gordura e a massa corporal predisseram $9 \%$ e $2 \%$, respectivamente, do desempenho da corrida de $30 \mathrm{~m}$ de jogadores adultos de handebol de ambos os sexos (Perez-Gomez et al., 2008). Ré et al. reportaram correlação significativa e inversa entre a velocidade de corrida de $30 \mathrm{~m}$ e a MC (0,30) em 286 jovens de 10 aos 16 anos de idade (Ré, 2005). Os autores consideraram que o aumento da massa corporal proporcionado por um maior acúmulo de gordura tem efeito negativo nos índices de desempenho motor. Entretanto, Guedes e Guedes observaram correlação significativa entre o desempenho no teste de corrida de $50 \mathrm{~m}$ e a $\mathrm{MC}$ (masculino $\mathrm{r}=$ 0,73; feminino $\mathrm{r}=0,55$ ) em escolares de sete aos 17 anos (Guedes e Guedes, 1993). As divergências entre os resultados encontrados podem estar ligadas as diferentes faixas etárias investigadas, pois correspondem a diferentes estágios de crescimento, desenvolvimento e maturação do aparelho locomotor.

O corrente estudo mostrou que parte significativa do desempenho em um teste que exige velocidade máxima de corrida pode ser atribuído as variáveis antropométricas medidas no presente estudo (62\%). Portanto, outras variáveis afetam o desempenho em corridas de curta distância. É importante ressaltar que o desempenho neste tipo de corrida depende não só da velocidade cíclica de movimento, mas também do tempo de reação e pela capacidade de aceleração (Hunter et al., 2004; Pilianidis et al., 2012). Parolis e Oliveira descrevem que 6\% do tempo para realizar a prova dos 100 metros rasos correspondem ao tempo de reação (1\%) e a saída do bloco (5\%) (Parolis, 2008). O tempo de reação é determinado pelo tempo gasto na identificação do estímulo, na seleção e programação da reposta (Schmidt, 2001), enquanto que a aceleração é determinada pela magnitude e frequência de impulsos contra o solo nos metros iniciais de corrida (Mero et al., 2006). Sendo assim, futuros estudos são necessários para avaliar a contribuição de outros parâmetros na velocidade em corrida de curta distância.

Concluindo, o presente estudo mostrou que a $\mathrm{DC}_{\mathrm{pa}}$ corresponde a $45 \%$, a estatura $33 \%$, a Cíbia $24 \%$ e a MC a $3 \%$ do desempenho na corrida de $50 \mathrm{~m}$. Estas variáveis antropométricas explicam aproximadamente $62 \%$ da VC. Em adição, os sujeitos com baixa velocidade apresentaram maior $\mathrm{MC}$, IMC e $\mathrm{DC}_{\mathrm{pa}}$. Por outro lado, os sujeitos com alta velocidade apresentaram maior estatura e $\mathrm{C}_{\text {tibia }}(\mathrm{p}<0,05)$, e a menor $\mathrm{DC}_{\mathrm{pa}}(\mathrm{p}<0,05)$. Portanto, pode-se concluir que as medidas antropométricas de baixo custo e fácil aplicação podem predizer de modo satisfatório o desempenho de velocidade de corrida de curta distância. No entanto, futuras investigações são necessárias para compreender os efeitos de outras variáveis antropométricas sobre a velocidade de corrida. Além disso, outras distâncias de corrida também devem ser testadas. 


\title{
WHAT IS THE CONTRIBUTION OF ANTHROPOMETRIC CHARACTERISTICS ON SPRINT RUN?
}

\begin{abstract}
The aim of this study was to determine the effects of anthropometric characteristics on sprint. It was measured the height, body mass (BM), body mass index (BMI), calf skin fold (CKF), tibia's length (TL) and running velocity $(50 \mathrm{~m})$ of 235 students. The multiple regression explain $62 \%$ of $50 \mathrm{~m}$ running performance $(\mathrm{p}<0.05)$. The simple linear regression showed that CKF represented $45 \%$, height $33 \%$, TL $24 \%$, and BM $3 \%$ of $50 \mathrm{~m}$ running performance $(\mathrm{p}<0.05)$. The cluster analyze showed that the low velocity group presented highest BM, BMI and CKF $(p<0,05)$, while the high velocity group presented the highest stature, TL and the lowest CKF $(\mathrm{p}<0,05)$. The results suggest that anthropometric variables used in the current study can significantly predict the $50 \mathrm{~m}$ sprint in young people.
\end{abstract}

Key words: Velocity. Running. Anthropometric. Performance.

\section{¿QUÉ ES LA CONTRIBUCIÓN LA CARACTERÍSTICAS ANTROPOMÉTRICAS EN LA CARRERA DE VELOCIDAD?}

\section{Resumen}

El estudio trata de evaluar los efectos de las características antropométricas en la carrera de velocidad. Fue evaluado la altura, masa corporal (MC), índice de masa corporal (IMC), pliegue cutáneo del pantorrilla $\left(\mathrm{DC}_{\mathrm{pa}}\right)$, longitud de la tibia $\left(\mathrm{C}_{\mathrm{t} \text { tía }}\right)$ y la carrera de velocidad $(50 \mathrm{~m})$ de 235 estudiantes. El analisis de regresión múltiple explica el $62 \%$ del rendimiento en la carrera $(\mathrm{p}<0,05)$. El análisis de

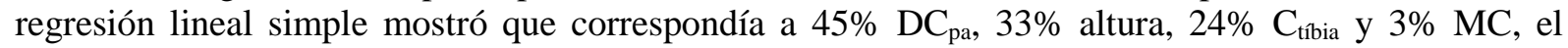
rendimiento en la carrera $(\mathrm{p}<0,05)$. El análisis de conglomerados mostró que el grupo de baja velocidad tuvo un mayor $\mathrm{MC}, \mathrm{DC}_{\mathrm{pa}}$ y el IMC $(\mathrm{p}<0,05)$, y el grupo de alta velocidad mostró una mayor altura, $\mathrm{C}_{\text {tibia }}$, e inferior $\mathrm{DC}_{\mathrm{pa}}(\mathrm{p}<0,05)$. Se concluye que las medidas antropométricas puede predecir el rendimiento de la carrera.

Palabras clave: Velocidad. Carrera. Antropometría. Desempeño.

\section{Referências}

BACK, T. W. The importance of a priori sample size Estimation in strength and conditioning Research. J Strength and Conditioning Research, v. 27, n. 8, p. 14, 2013.

COH, M.; MILANOVIC, D.; KAMPMILLER, T. Morphologic and kinematic characteristics of elite sprinters. Collegium Antropologicum, v. 25, n. 2, p. 605-610, Dec 2001. ISSN 03506134. Disponível em: <<Go to ISI $>/ /$ WOS:000173295100023 >.

GRAUBNER, R. N., E. Biomechanical Analysis of the Sprint and Hurdles Events at the 2009 IAAF World Championships in Athletics. New Studies in Athletics., v. 26, 2011.

GUEDES, D. P.; GUEDES, J. E. [Growth and motor performance of schoolchildren from the city of Londrina, Parana, Brazil]. Cad Saude Publica, v. 9 Suppl 1, p. 58-70, 1993. ISSN 0102-311X (Print)

0102-311X (Linking). Disponível em: < http://www.ncbi.nlm.nih.gov/pubmed/15448821 >.

HUNTER, J. P.; MARSHALL, R. N.; MCNAIR, P. J. Interaction of step length and step rate during sprint running. Med Sci Sports Exerc, v. 36, n. 2, p. 261-71, Feb 2004. ISSN 01959131 (Print)

0195-9131 (Linking). Disponível em: < http://www.ncbi.nlm.nih.gov/pubmed/14767249 >. 
MACKALA, K. Optimization of performance through kinematic analysis of the different phases of the 100 meters. New Studies in Athletics., v. 22, n. 2, 2007.

MACKALA, K.; FOSTIAK, M.; KOWALSKI, K. Selected Determinants of Acceleration in the 100m Sprint. Journal of Human Kinetics, v. 45, n. 1, p. 135-148, Mar 2015. ISSN 16405544. Disponível em: <<Go to ISI>://WOS:000353159100014 >.

MACKAŁA, K. M., A. A Kinematics analysis of three best 100m performances. Ever J Hum Kinetics, v. 36, p. 11, 2013.

MERO, A. et al. Effects of muscle-tendon length on joint moment and power during sprint starts. Journal of Sports Sciences, v. 24, n. 2, p. 165-173, Feb 2006. ISSN 0264-0414. Disponível em: <<Go to ISI >:/WOS:000235290400008 >.

NAGANO, A.; KOMURA, T. Longer moment arm results in smaller joint moment development, power and work outputs in fast motions. Journal of Biomechanics, v. 36, n. 11, p. 1675-1681, Nov 2003. ISSN 0021-9290. Disponível em: < <Go to ISI > //WOS:000185956500011 >.

PAROLIS, S. C. O., P.R. Atletismo: velocidade máxima de deslocamento na corrida de 100 metros rasos: um estudo de caso. Conexões v. 6, p. 11, 2008.

PEREZ-GOMEZ, J. et al. Role of muscle mass on sprint performance: gender differences? Eur J Appl Physiol, v. 102, n. 6, p. 685-94, Apr 2008. ISSN 1439-6319 (Print) 1439-6319 (Linking). Disponível em: < http://www.ncbi.nlm.nih.gov/pubmed/18084774 >.

PILIANIDIS, T. et al. Start Reaction Time and Performance at the Sprint Events in the Olympic Games. Kinesiology, v. 44, n. 1, p. 67-72, Jun 2012. ISSN 1331-1441. Disponível em: <<Go to ISI>://WOS:000306676900009>.

RÉ, H. B., L.P.; TEIXEIRA, C.P.; BOHME, M.T.S. Relações entre crescimento, desenvolvimento motor, maturação biológica e idade cronológica em jovens do sexo masculino. Rev Bras Educ Fís Esporte, v. 19, n. 2, p. 9, 2005.

ROSS, A.; LEVERITT, M. Long-term metabolic and skeletal muscle adaptations to shortsprint training: implications for sprint training and tapering. Sports Med, v. 31, n. 15, p. 1063-82, 2001. ISSN 0112-1642 (Print)

0112-1642 (Linking). Disponível em: < http://www.ncbi.nlm.nih.gov/pubmed/11735686 >.

ROSS, A.; LEVERITT, M.; RIEK, S. Neural influences on sprint running: training adaptations and acute responses. Sports Med, v. 31, n. 6, p. 409-25, 2001. ISSN 0112-1642 (Print)

0112-1642 (Linking). Disponível em: < http://www.ncbi.nlm.nih.gov/pubmed/11394561 >.

SCHMIDT, R. A. W., C.A. Aprendizagem e performance motora: uma abordagem da aprendizagem baseada no problema. Porto Alegre: Artmed, 2001. 
THOMAS, S.; READING, J.; SHEPHARD, R. J. Revision of the Physical Activity Readiness Questionnaire (PAR-Q). Can J Sport Sci, v. 17, n. 4, p. 338-45, Dec 1992. ISSN 0833-1235 (Print)

0833-1235 (Linking). Disponível em: < http://www.ncbi.nlm.nih.gov/pubmed/1330274 >.

Recebido em: 12/04/2016

Revisado em: 17/06/2016

Aprovado em: 17/06/2016

Endereço para correspondência:

vieiraca1@uol.com.br

Carlos Alexandre Vieira

Universidade Federal de Goiás, Faculdade de Educação Física.

Campus Samambaia

Setor Samambaia

74001970 - Goiânia, GO - Brasil 